\title{
Fuzzy Logic based Short-Term Electricity Demand Forecast
}

\author{
P.Lakshmi Priya ${ }^{\# 1}$, V.S.Felix Enigo ${ }^{\# 2}$ \\ \# Department of Computer Science and Engineering, \\ SSN College of Engineering, Chennai, Tamil Nadu, India \\ ${ }^{1}$ priyalakshmi1994@gmail.com \\ ${ }^{2}$ felixvs@ssn.edu.in
}

\begin{abstract}
Short-term demand forecasting is crucial for the operations of planning and scheduling of an electricity power generation. Since short-term load patterns are non-linear, approaches such as neural network, fuzzy system and genetic algorithms are proven more effective. Neural Networks and genetic approaches have some limitations such as black box learning approach, not much useful when it function as standalone (mostly used with neural network to find optimal parameters) and respectively. Fuzzy systems use comprehensible rules as prior knowledge to get sensible results, but it lacks selflearning. In this paper, we have proved that by fine tuning various parameters and varying suitable methods of a fuzzy system, it is possible to get accuracy as close to that of neural network, with the additional benefit of results with reasoning.
\end{abstract}

Keywords - Forecasting, Fuzzy Inference System, Neural Network

\section{INTRODUCTION}

The modern techniques of artificial intelligence have found application in almost all the fields that involve human knowledge. However, great emphasis is given to the areas that require accurate measurements such as predicting future values, identifying hidden patterns and classification problems. One such application which is most essential for humans in today's world is electricity and its generation. It has become a part of modern life. Since electricity is a scarce resource, it should be optimally generated and used. Electricity cannot be stored, rather it is generated on demand. Hence, careful forecast of electricity demand is crucial. Compare to long term electricity demand, short term demand prediction is imperative as it directly impacts the next day or next hour need for homes and industries. Insufficient electricity generation paralyses entire society comprising businesses, industries and home. Hence forth, forecasting short term electricity demand is important for future electricity generation. It helps to plan, budget, schedule maintenance, thereby reducing the overall cost. Short term electricity forecast avoids shortage in power and overcapacity that may leads to wastage of power. Short term forecast refers to a time period that spans from hour to a week [1], medium term refers to duration from a week to a year and long term prediction is done for more than a year.

The approaches for load forecasting are broadly classified as traditional methods and soft computing methods. Traditional methods uses statistical approach for time series analysis of data using regression, which finds the causal relationships between a dependent and one or more independent variables that, can be estimated [2]. Autoregressive Moving Average (ARMA) [3] and Autoregressive Integrated Moving Average (ARIMA) are some of the variants of regression analysis. ARMA predicts the future load based on the past values and its error. Whereas ARIMA predicts linear changes in load data such as trends which will be either increasing or decreasing with respect to time. Such methods cannot be applied over non-linear data for prediction. Among soft computing approaches, neural networks are commonly used for load prediction due to its applicability for non-linear data and its accuracy. But the limitations of neural network is that though it has the ability to learn by itself the kind of data given as input, the learning process is a black box [4] i.e. it is not possible to infer how it arrives at that output. Genetic algorithms are used in electricity demand forecasting [5] mostly along with neural networks rather as standalone approach. It is used to improve the performance of the system by obtaining the optimal parameters that enables quick convergence of the system and for better accuracy. Fuzzy based approaches [6] apply mathematic calculus to translate the subjective human knowledge about the real processes which are expressed as If-then rules to get an understandable output. But, it lacks ability to learn by itself as in neural networks.

In this paper, we show that using fuzzy based load prediction it is possible to get the load prediction accuracy as close to neural networks by carefully tuning of parameters as well as methods used in each step of fuzzy processing. Fuzzy based reasoning is characterized by generalizing classical logic for reasoning under nonlinear and uncertain conditions. It is the most appropriate method for describing the human knowledge of enormous data, in our case the data refers to electricity load data. There are many uncertain parameters, which affects the electricity load forecasting such as weather conditions (temperature, rainfall, humidity), time factor (short term, midterm, long term), technology, population growth, calendar effects (seasons, weekday, holiday, special day), 
and customer factor (irregularity in usage) [7]. Fuzzy system identifies the electricity load pattern and predicts the future short term electricity demand.

\section{UNDERLYING TECHNOLOGY}

Fuzzy logic is a type of reasoning that can categorize the input over a range, apart from binary true or false. It works similar to the manner in which our human brain functions. It manipulates practical knowledge with some level of uncertainty. The fuzzy logic system is described through fuzzy sets and fuzzy rules. A fuzzy set is defined by the degree of membership of the variable. Each fuzzy set corresponds to a linguistic concept such as high, very high, medium, low etc. representing its numeric values [7]. A fuzzy rule is written as antecedent and consequence that represents the combination of fuzzy sets and its conclusion respectively.

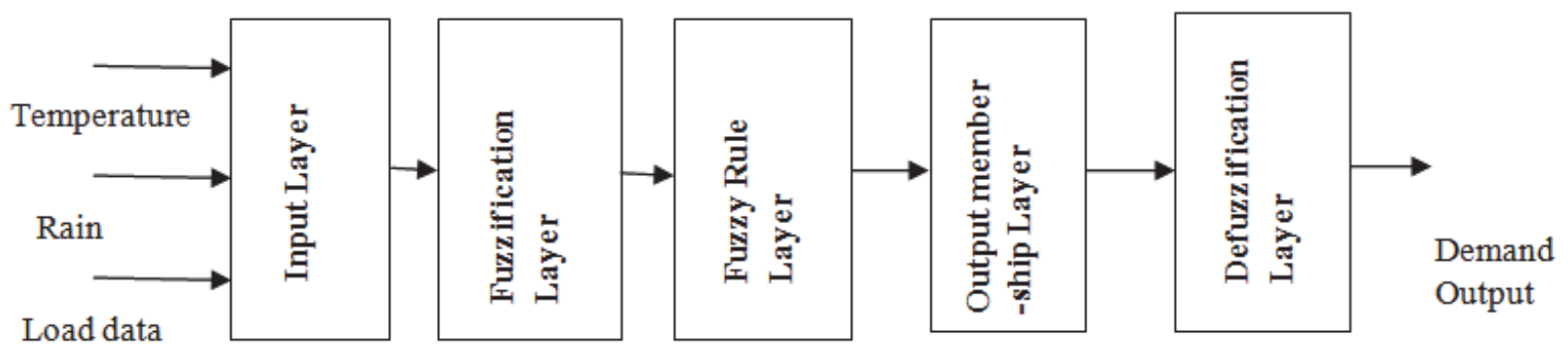

Fig. 1. Process of Fuzzy Inference System

A fuzzy inference system has three stages. The first stage, maps the numerical inputs to the degree of membership of the corresponding fuzzy sets. This process is termed as fuzzification. In the second stage, fuzzy processing is performed based on the fuzzy rules over the given input data. The final stage is the de-fuzzification process in which the aggregated fuzzy values are mapped to crisp values using membership functions. Some of the most commonly used methods for de-fuzzification are:[8]

a) Centroid of Gravity or Centroid of Area

b) Bisector of Area

c) Smallest of Maximum

d) Largest of Maximum

e) Mean of Maximum

In Centroid of Area, the fuzzified aggregate set is converted to a crisp value based on the center of gravity. Bisector of Area partitions the aggregated set into two regions with same area and the line of bisector corresponds to the crisp value. Smallest of maximum determines the smallest value of the region that has maximum membership value. Whereas, Largest of maximum is similar to the smallest of maximum, but here it takes the largest value of the region to compute the crisp value. Mean of Maximum method takes the mean value when more than one element has maximum membership values for computing single value.

\section{SYSTEM IMPLEMENTATION}

The proposed system is implemented using Fuzzy Inference System. The objective of this work is to tune the parameters to obtain accuracy as close to neural approach with understandable results. To conduct the experiment, we collected electricity demand dataset from Tamil Nadu Electricity Board (TNEB), the rainfall data is taken from the meteorological website of Department of Geography and the temperature data is extracted from wunderground website. To automate the extraction process, shell scripts are used to pull out the data from the website. The collected data from all sources are populated into a single dataset. This dataset serves as an input to the fuzzy inference system.

In the first step, we have conducted various runs on fuzzy system, by varying the intervals of the input, so that appropriate membership functions can be used. There are different types of membership functions such as triangular, trapezoidal, Gaussian, sigmoid etc [10]. Depending upon the problem size and type any one can be chosen. In our proposed system, we found better accuracy with respect to triangular membership function in predicting the electricity demand. The triangular membership function is defined by a lower limit a, an upper limit $\mathrm{b}$ and a value $\mathrm{m}$, where $\mathrm{a}<\mathrm{m}<\mathrm{b}$. In our case, we have taken five input parameters such as temperature, rainfall, Day_type, Season_type and historical_electricity_demand for predicting electricity demand. Each of these parameters are mapped to three intervals Low, Medium and High as shown in Figure 2. 

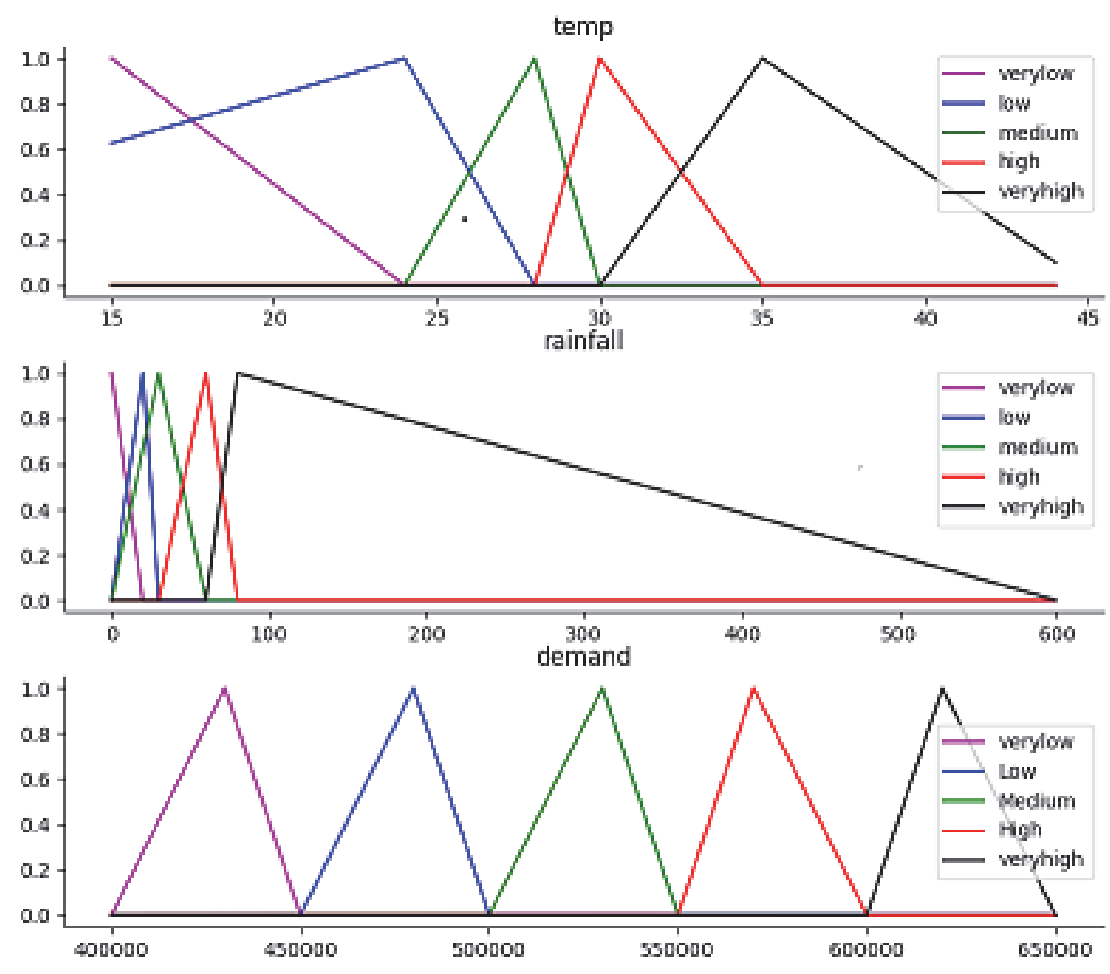

Fig. 2. Process of Fuzzification

Further, we have divided our dataset into three seasons. April to June is treated as summer season, December to March as winter season and July to September as rainy season. And for each season, fuzzy logic is applied and electricity demand is predicted separately. This is done to improve the prediction accuracy, since for each season the baseline demand will be different.

After selecting optimal membership function that suits the dataset, the next step is to construct fuzzy rules based on the membership functions. For fuzzy rule construction, two types of fuzzy inference models can be used. It could be either mamdani or Sugeno type based on the type of output required. In Mamdani fuzzy inference type, the output will be of fuzzy set [11].

E.g. If temperature is low and rainfall is high, then electricity demand is low

Whereas, Takagi Sugeno type fuzzy inference system gives the output as a linear function.

E.g. If temperature is low and rainfall is high, then electricity demand is axl $+b x 2+c$

Where $\mathrm{a}, \mathrm{b}$ and $\mathrm{c}$ are constants, $\mathrm{x} 1$ and $\mathrm{x} 2$ are input parameters (e.g. temperature, rainfall).

In our work, we have used sugeno type inference system as we need the electricity demand forecast in numeric values rather than fuzzy set. Next, the outputs from each rule which are fuzzy sets are combined using aggregation operators to produce a single fuzzy set. Minimum and maximum are the basic aggregation operators in fuzzy logic and it satisfies certain axioms (monotonic, symmetric, associative and idempotent) while aggregating the membership functions [11]. The minimum and maximum gives the smallest and largest value in the fuzzy set respectively, which is shown in the Figure 3.

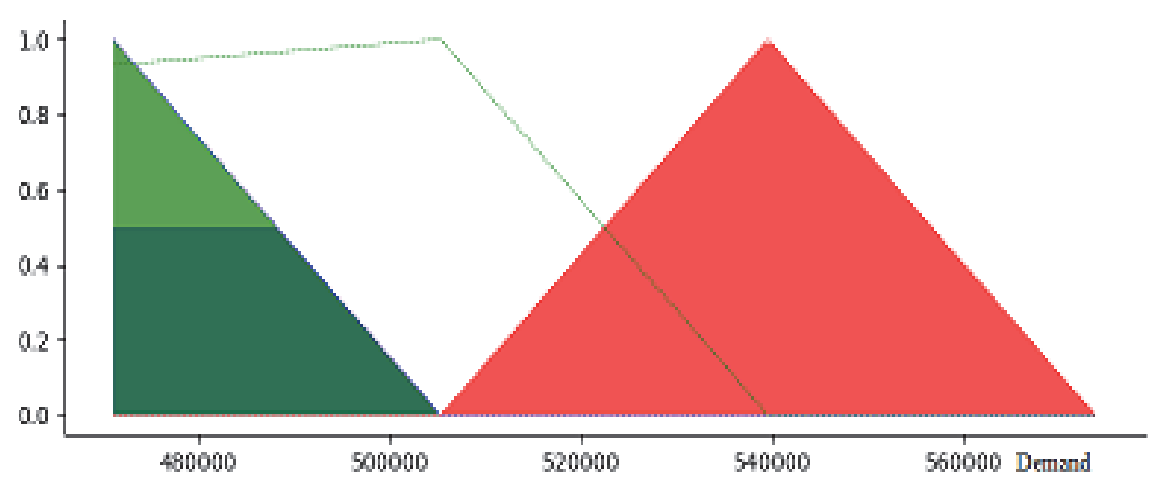

Fig. 3. Input mapped to membership values 
The final step in fuzzy inference system is the de-fuzzification process. It converts the single aggregated output to a crisp value by applying various de-fuzzification methods [12]. The most common de-fuzzification methods are i) Center of sums ii) Center of gravity or Centroid of Area iii) Center of Area or Bisector of Area iv) Weighted Average v) Maxima Methods. One of the aforementioned methods is selected depending upon the application requirements. The mean and maximum methods are not applicable for our data as we require accurate predicted value. So, we have chosen Centroid of Area method that divides the aggregated output into two equal masses rather than maximum or mean value of aggregated output. Here, centroid of the area indicates the predicted electricity demand as crisp value which is shown in Figure 4.

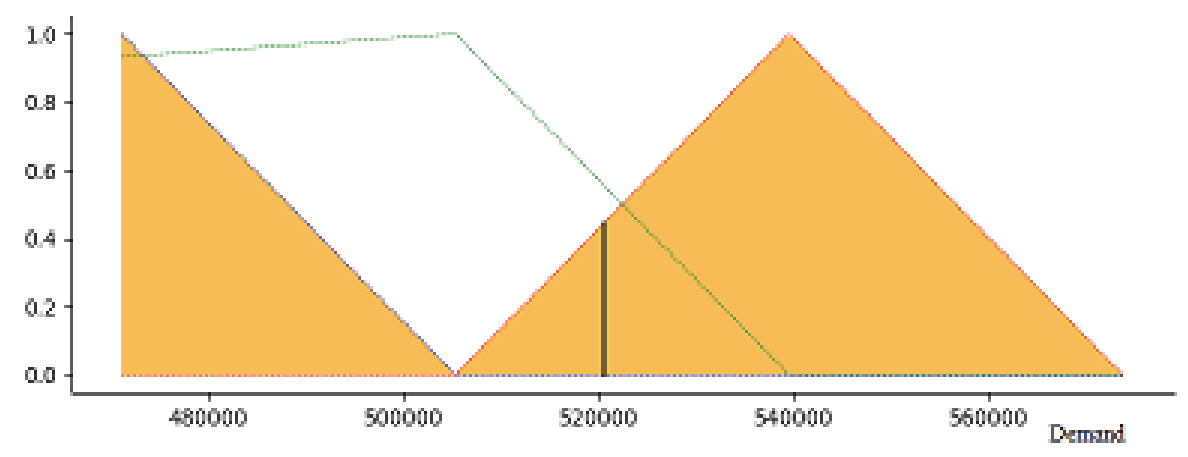

Fig. 4. Defuzzification of aggregated fuzzy set

\section{RESULTS AND DISCUSSION}

In this paper, performance improvements are achieved by tuning various parameters and varying the methods based on the dataset to obtain maximum accuracy for short term electricity demand forecast. The obtained results of fuzzy system are compared with the prediction accuracy of neural network to see how close the results are. Two performance measures i) Absolute Percentage Error (APE) and ii) Mean APE (MAPE) is used to verify the performance of the fuzzy system.

APE is a value that represents the difference between the predicted value of the model and the actual value. Its value ranges from zero to infinity. A low APE is desired for a good model i.e. the predicted results should be close to the actual value. It is computed using the below formula:

$$
A P E=\left(\frac{x-y}{x}\right) * 100
$$

where, $\mathrm{x}$ is the actual value and $\mathrm{y}$ is the predicted value.

Mean APE (MAPE) is the mean of APE and it is a used to compute the overall accuracy of the system. It is computed as follows:

$$
\text { MAPE }=\left(\frac{1}{n} * \frac{\sum x-y}{x}\right) * 100
$$

First we varied different types of membership functions over the data to find the impact on the accuracy and we obtained the results shown in Table1. From the table, it is inferred that for the given dataset, triangular functions perform better than other membership functions.

Table 1. APE for different membership functions

\begin{tabular}{|c|c|c|c|c|c|c|}
\hline Temperature & Rainfall & Actual Demand & $\begin{array}{c}\text { Sigmoid } \\
\text { Function }\end{array}$ & $\begin{array}{c}\text { Trapizoidal } \\
\text { Function }\end{array}$ & $\begin{array}{c}\text { Triangular } \\
\text { Function }\end{array}$ & $\begin{array}{c}\text { Gaussian } \\
\text { Function }\end{array}$ \\
\hline 25 & 0 & 498519 & 5.56 & 6.98 & 4.53 & 6.03 \\
\hline 26 & 7 & 514631 & 3.08 & 5.21 & 6.04 & 6.98 \\
\hline 26 & 78 & 521382 & 5.89 & 5.63 & 4.00 & 4.67 \\
\hline 25 & 34 & 505629 & 4.00 & 7.56 & 3.31 & 4.09 \\
\hline 26 & 0 & 519049 & 4.87 & 8.98 & 4.71 & 5.67 \\
\hline 25 & 11 & 523754 & 4.99 & 6.00 & 5.26 & 5.09 \\
\hline 24 & 23 & 542991 & 5.67 & 7.83 & 4.20 & 5.23 \\
\hline 34 & 0 & 613490 & 4.45 & 3.28 & 3.83 & 3.98 \\
\hline 33 & 0 & 603854 & 2.09 & 3.11 & 2.41 & 3.02 \\
\hline & & MAPE $=$ & $4.06(\%)$ & $5.44(\%)$ & $3.82(\%)$ & $4.46(\%)$ \\
\hline
\end{tabular}


Next, different types of de-fuzzification methods are applied to make a better prediction of electricity demand. As said earlier, mean and maximum methods are not applicable to our data. Hence, we applied Centroid of Area and Bisector of Area to the dataset and found that centroid of area is performing better compare to bisector approach. This is because, Centroid of Area divides the aggregated output into two equal masses rather than equal area as in bisector method. Based on the above analysis, we found that the combination of triangular membership function with Centroid of Area de-fuzzification gives better performance than other combinations which is shown in Table 2.

Table 2. APE for different defuzzification methods

\begin{tabular}{|c|c|c|c|c|c|c|}
\hline Temperature & Rainfall & Actual Demand & $\begin{array}{c}\text { Predicted } \\
\text { Bisector }\end{array}$ & APE(\%) & $\begin{array}{c}\text { Predicted } \\
\text { Centroid }\end{array}$ & APE(\%) \\
\hline 25 & 0 & 498519 & 464198 & 6.88 & 465933 & 6.53 \\
\hline 26 & 7 & 514631 & 463784 & 9.80 & 483512 & 6.04 \\
\hline 26 & 78 & 521382 & 472568 & 9.36 & 490095 & 6.00 \\
\hline 25 & 34 & 505629 & 473595 & 6.33 & 488843 & 3.31 \\
\hline 26 & 0 & 519049 & 493586 & 4.90 & 484198 & 6.71 \\
\hline 25 & 11 & 523754 & 472365 & 9.81 & 496190 & 5.26 \\
\hline 24 & 23 & 542991 & 495623 & 8.72 & 498449 & 8.20 \\
\hline 34 & 0 & 613490 & 543568 & 11.3 & 590643 & 3.83 \\
\hline 33 & 0 & 603854 & 553625 & 8.31 & 608709 & 2.41 \\
\hline & \multicolumn{7}{r}{ MAPE $=$} & $7.54(\%)$ & & $4.82(\%)$ \\
\hline
\end{tabular}

Finally, we compared the accuracy of the fuzzy system with tuned parameter and optimal de-fuzzification method with that of neural network. The MAPE of fuzzy system is found to be $3.17 \%$ and that of neural network $3.12 \%$ which is almost same. Thus, we have shown that by fine tuning various parameters of fuzzy system, it is possible to get accuracy as close to that of neural network, with the additional benefit of results with reasoning which is a black box in neural network.

Table 3. Comparision of Fuzzy Logic and Neural Network

\begin{tabular}{|c|c|c|c|c|c|c|}
\hline Temperature & Rainfall & Actual Demand & $\begin{array}{c}\text { Predicted } \\
\text { Fuzzy }\end{array}$ & APE(\%) & $\begin{array}{c}\text { Predicted } \\
\text { Neural }\end{array}$ & APE(\%) \\
\hline 25 & 0 & 498519 & 464198 & 6.53 & 515552 & 3.41 \\
\hline 26 & 7 & 514631 & 463784 & 6.04 & 507612 & 1.36 \\
\hline 26 & 78 & 521382 & 472568 & 6.00 & 488711 & 6.26 \\
\hline 25 & 34 & 505629 & 473595 & 3.31 & 496057 & 1.89 \\
\hline 26 & 0 & 519049 & 493586 & 6.71 & 489473 & 5.69 \\
\hline 25 & 11 & 523754 & 472365 & 5.26 & 491300 & 5.19 \\
\hline 24 & 23 & 542991 & 495623 & 8.20 & 539003 & 2.04 \\
\hline 34 & 0 & 613490 & 543568 & 3.83 & 543211 & 4.96 \\
\hline 33 & 0 & 603854 & 553625 & 2.41 & 598861 & 2.04 \\
\hline & & MAPE $=$ & $3.17(\%)$ & & $3.12(\%)$ \\
\hline
\end{tabular}

\section{CONCLUSION}

Load Forecasting plays a crucial role in all aspects of planning, operation, and control in electricity generation and distribution. Neural networks and fuzzy system are widely used techniques for prediction in nonlinear data. Though neural networks are found to produce better accuracy than fuzzy based system, the learning procedure of neural networks are black box. While fuzzy logic, models the inference that mimics human brain using simple If-Then rules, but lacks self-learning. In this paper, we have overcome this disability by carefully tuning the parameters and methods that implements the fuzzy logic to obtain the accuracy of $96.83 \%$ which nearly equal to the neural networks which produced $96.88 \%$.

In future, we intend to implement electricity load prediction using various types of neuro-fuzzy architectures that combines the advantages of both neural and fuzzy system to further improve the prediction accuracy. 


\section{REFERENCES}

[1] K.Navjot and K.Amrit, Predictive Modelling Approach to Data Mining for forecasting electricity consumption, 6th International Conference-Cloud System and Big Data Engineering, Vol. 3, pp. 724- 740, 2014.

[2] P.M.Patel and P.S. Ashish, Fuzzy logic methodology for short term load forecasting, International Journal of Research in Engiineering and Technology, Vol. 3, pp: 2321- 7308, 2016.

[3] K.Jagbir, Short-term Load Forecasting using Fuzzy Logic of 220kV Transmission Line, International Journal of Engineering Research and Technology, Vol. 3, pp: 2278-0181,2015.

[4] Arunesh Kumar Singh, S.Ibraheem, Khatoon and Md.Muazzam, An Overview of Electricity Demand Forecasting Techniques,National Conference on Emerging Trends in Electrical, Instrumentation \& Communication Engineering, Vol. 3, No.3,2013.

[5] A.Saima, Empirical Comparison of Prediction Methods for Electricity Consumption Forecasting, IEEE Transactions on Power System, Vol. 10,2014

[6] H.Vinutha, Electric Load Forecasting using a Neural Network Approach, International Journal of Computer Trends and Technology (IJCTT) - volume 11 No.6,2016.

[7] H.Hasan and C.Mehmet, A Fuzzy Logic Based Short-term Load Forecast for the Holidays, International Journal of Machine Learning and Computing,Vol. 6, pp: 57,2013.

[8] M.Mordjaoui, Short term electric load, Conference on nonlinear science and complexity, Ankara, Turkey, vol.6, pp. 12-32,2016.

[9] P.K.Dash, S.Dash,S.Rama Keishna and S.Rahman Dash, Building a Fuzzy Expert System for Electric Load Forecasting Using a Hybrid Neural Network, International Conference on Expert Systems With Applications, Vol. 9, pp: 407-421,2014.

[10] K.Nikola and Kasabov, Network and Fuzzy Logic, LNCS, MIT Press,Springer ,1998.

[11] K.M.Motahar Hossain, On Appropriate Selection of Fuzzy Aggregation Operators in Medical Decision Support System, conference on data science,vol.3,2012.

[12] N.Sameena, A.Afshar and B.Ranjit, Effect of Different Defuzzification Method in a Fuzzy based Load Balancing Application, International Journal of Computer Science Issues, Vol. 8, No 1, pp:261-267, 2011. 